If we believe the origin of the poison to be in the drain, vault, cesspool or soiled earth, we are most likely to disregard the fact that the sick person is the source of an infinite multiplication of the poison. I would not detract a particle from the value of cleanliness or the necessity of care and treatment of all foul matters, but it seems to me that we should, if possible, recognize the true source of the enemy and direct our energies to the best advantage.

What are the most effective means for preventing the spread of these diseases? I believe that with almost universal consent of sanitarians we may say, absolute isolation of all cases, and the disinfection of everything that has become infected by a case.

The best form of isolation is without doubt in hospitals where the contagion may be concentrated and all means of communication between well and sick can be kept under control.

I would remove all such cases to a hospital except where proper isolation could be secured at home.

We have had an example in Boston, of what may be done with a contagious disease when the power and facilities for isolation are given.

We can but hope that the day is not far distant when we shall have the facilities for isolating diphtheria, scarlet fever, measles and many other contagious disease.

Can we depend upon disinfectants for the absolute destruction of these disease-germs, and.if so, what are they?

The first question is well answered by Dr. Sternberg in a prize essay a few weeks ago, as follows :

"Evidence of disinfection may be obtained, 1st. From the practical experiments and experience of those engaged in sanitary work; 2d. By inoculation experiments upon susceptible animals; and $3 d$. By experiments made directly upon known disease-germs."

I think there can be no reasonable doubt as to the power of certain disinfectants to kill all living microorganisms or disease germs. I believe that all bacteriologists agree as to this fact, but find a great difference in the power of some germs over others to retain life in the presence of the disinfectants.

'The germ containing the spore is found to live where the germs without spores are readily killed.

According to Sternberg, there is no reason to believe that spores exist in the germs of cholera. smallpox, scarlet fever, diphtheria or yellow fever.

The agents found by experiment to be most useful in destroying disease germs containing spores are, fire, steam under pressure, boiling in water, chloride of lime and bichloride of mercury, and for those not containing spores, fire, boiling in water, dry heat, chloride of lime, solution of chlorinated soda, bichloride of mercury, sulphurous acid gas, carbolic acid, sulphate of copper and chloride of zinc.

Practically, it will be found best to destroy by fire such articles as are too badly soiled or worn to be of value; everything that may be wet without injury should be boiled in water for at least one hour; articles which cannot be wet without injury should be fumigated with burning sulphur, or subjected to the action of superheated steam. Three or four pounds of sulphur to each 1,000 cubic feet of space should be burned as rapidly as possible with the room tightly closed, and all articles therein so scattered and hung about as to permit easy access of the gas. The room should remain closed at least six hours.
To disinfect by steam, the articles should be hung about in the tightly closed room, and the temperature raised to $230^{\circ} \mathrm{F}$., and held at that point for ten minutes, or $220^{\circ} \mathrm{F}$. for fifteen minutes.

Floors and other hard surfaces may be washed with a solution of mercuric chloride (one drachm to the gallon of water) or a solution of the fresh chloride of lime, four ounces to the gallon of water. Nothing should be carried from the sick room before being disinfected. Articles which can be wet may be placed in one of the above solutions, kept in a wooden receptacle until such time as they can be removed for boiling.

All discharges from the bowels, bladder or stomach of patients suffering from typhoid fever, cholera or yellow fever should be received in vessels containing one of the above solutions in quantity greater than the infective material itself, and thoroughly mixed before being disposed of.

Gargling or spraying the diphtheretic throat frequently with an active disinfectant and frequent bathing or oiling the skin of the scarlatinal patient may measurably prevent the escape and dissemination of the poison.

\section{SUBLUXATION OF THE RADIAL HEAD IN CHILDREN.1}

BY H. W. CUShING, M.D.,

Surgeon to Out-Patients, Carney Hospital.

THE cases to be reported, seen soon after the publication of Mr. Hutchinson's article on this subject, illustrate very well the lesion described by him, and furnish examples of an injury producing an obscure, and, at times, puzzling group of symptoms.

An accurate diagnosis of injuries to the elbow joint, especially in children, is often a difficult problem to solve, and any addition to the surgeon's knowledge of these lesions, which will enable him to diminish the number of cases he is forced to class under the ofttimes unsatisfactory diagnosis of "sprain of elbow," is gladly welcomed. And this lesion appears to be a correct explanation of the group of symptoms about to be described.

The injury is apparently much oftener met with than the brief mention of it in surgical text-books would lead one to suppose. A large proportion of cases are patients five years of age, or under. Hutchinson states that he was unable to produce it experimentally in subjects over five or six years. Lindeman, ${ }^{8}$ in his report of twenty-four cases, finds all under five years, and a majority between nine months and two years.

In most cases it is the result of a sudden traction on the extended upper extremity, such as a child receives who, led by the hand, stumbles, and is prevented from falling by the jerk with which its nurse restores it to an upright position. The same conditions obtain when a child is dragged along by the hand, as is frequently seen, or lifted over an obstacle, or into a chair or vehicle. 'This explains why so many of these cases are found in children under five years of age, since this is a period of life during which the custom of lifting or leading children by the hand causes frequent 1 Read before the Boston Society for Med:cal Observation, January
4th, 1886.
2 Annais of Surgery, August, 1885, J. Hutchinson, Jr., F.K.C.S.
3 British Medical Journal, December 5, 1885, Sydney H. Lindemen,
M.R.C.S. 
exposure of weak and incompletely developed articular structures to violence of the nature just described. This lesion is also said to result from a blow or fall received on the posterior surface of the semi-flexed elbow, which forces the head of the radius directly forward, but this is probably of rare occurrence.

Opinions differ as to the manner in which this lesion is produced. The sudden force applied to the fore-arm in these cases causes a displacement of the radius downward, so that its head is withdrawn from the orbicular ligament to a greater or less extent.

Mr. Hutchinson's article contains the only report of a dissection of this lesion that $I$ have found, and is briefly as follows. He calls attention to the shape of the radial head; and to the difference in depth and contour of the radial and uluar sides, the former of which is a rounded surface, while the latter is rectangular in outline.

These surfaces and angles, he concludes, play an important part in replacing the head of the radius within the orbicular ligament by their change in position during supination and pronation. He found by experiment that in subjects under five or six years of age, forcible traction during supination causes a slipping up of the orbicular ligament, with or without rupture of the suborbicular membrane or capsule. This was demonstrated by dissection, and he believes that the lesion consists, in most cases, in an escape of the head from the grasp of the orbicular ligament. In these cases, where the force is great, there is also probably a rupture of that part of the capsule connecting this ligament to the neck of the radius. As shown by his figure, the dislocation is a partial one, and the radius is still more or less in contact with the lesser sigmoid cavity.

Lindeman thinks the tendon of the biceps, one of whose actions is flexion and supination of the forearm, is an important factor in causing the displacement, and pulls the radius forward out of its position.

The anatomical arrangement of the articulation seems to indicate that the lesion is produced as follows : The force applied at the wrist by traction on the hand affects the radius mainly, since that bone occupies a greater portion of this joint, and is transmitted through it to the elbow, where the radial head is held in place by a group of ligaments, of which the anterior and external lateral act as guys to the orbicular, which in turn, holds the radius in its normal position. The orbicular is itself attached to the radial neck, and this is the weakest point in the structure. In the position of the joint in which the accident occurs, the posterior fibres of the external lateral ligament are relaxed, and the tense anterior ligament, with possibly the anterior fibres of the external lateral, is the one which is left to support the orbicular against the traction from below, which it is strong enough to do. The force is thus directed to the insertion of the orbicular about the radial neck, at the anterior portion of its circumference; this yields, and a subluxation is the result.

The direction forward which the radial head almost invariably takes, is probably due to the yielding of the ligament at this point, and possibly also to the traction of the bicipital tendon. The extent of displacement varies with the elasticity of the tissue, and the rupture or non-rupture of the orbicular insertion. The rupture occurs anteriorly, since, as just stated, the anterior ligamentous attachments are tense in this position, and the force is exerted here first, while the posterior structures are relaxed. This change of position of the radius causes the traction to be transmitted to other structures, and further injury is avoided. The weakness and elasticity of infantile tissues is probably the explanation why this lesion is so uncommon in adults, where such mobility of osseous structure is absent, and such an injury would cause a " sprain" only. The clinical history is that the patient is injured in a majority of cases, as above described. Often a click or thud is noticed, or something is felt to give way, by the person holding the child's hand. The patient cries with pain, and the arm drops motionless to the side, with the elbow semi-flexed, and the hand partly pronated. The attendant is frequently puzzled to determine whether the wrist, elbow, or shoulder is the seat of the trouble, the latter being often designated as the location of the lesion. If the subluxation is reduced, either spontaneously or unconsciously by the parent during passive motion or other manipulation, all symptoms suddenly vanish, and nothing remains except a slight tenderness over the radial head, which soon disappears. If unreduced, the pain and impairment of motion persist, and medical aid is sought.

From a physical examination of the patient the surgeon usually obtains the following data: The injured arm hangs motionless by the side, with the elbow somewhat flexed, and hand semi-pronated. Attempts to use the latter, cause marked pain. Surface changes are usually absent, unless the accident was of such a nature as to cause additional lesions. No deformity exists except when the subluxation is marked, in which case the projection of the radial head causes a slight prominence below the external condyle on the anterior surface of the elbow. Tenderness over the head of the radius is the most constant symptom. In some cases, an increased surface temperature exists, with slight swelling. By 'careful manipulation, the projecting edge of the radial head can be felt, but it is easily overlooked, and the joint appears perfectly normal so far as its anatomical details are concerued. In some cases, flexion and extension are painful, but usually supination only causes discomfort. In most cases, supination alone is limited apparently by a mechanical obstacle. If not reduced by the above manipulation, the arm drops back into its former position, a symptom considered by Goyrand as pathognomonic. ${ }^{4}$ Limitation of other movements of the elbow, if present, is due to muscular resistance.

If untreated, or the affected articulation is imprudently used, the pain and tenderness become more marked, and it is not improbable that the commencing periostitis or synovitis may transform what was originally a simple injury into a lesion, which results in the destruction of a joint. When the intimate relation of the injured tissues to the periosteum of the radius and the synovial membrane of the elbow is considered, this seems quite possible, and, as Mr. Hutchinson suggests, this may explain the mysterious origin of many cases of chronic arthritis of the elbow in children.

The rapidity with which all symptoms vanish after reduction, a thing which frequently happens unexpectedly during examination, is a striking peculiarity of these cases.

The diagnosis lies between sprain and dislocation, other injuries to the elbow joint being easily elimi4 Boston Medical and Surgical Journal, September 18, 1862, R. M.
Hodges, M.D. 
nated, and the weight of opinion being opposed to Goyrand's theory that the symptoms are due to a displacement of the interarticular fibro-cartilage of the wrist in front of the carpal extremity of the ulnar. ${ }^{5}$

The diagnostic points are: the source of the injury; the localized tenderness; the limitation of supination only, and the rapid relief following reduction. The rotation of its head is apt to give the impression that the radius is in its normal position, and the surgeon is at a loss to account for the limitation of supination, which is almost the only abnormality discovered. And his perplexity is not at all diminished whon he finds that suddenly all symptoms have disappeared as if by magic, leaving no trace of their previous existence.

The prognosis is generally good if the reduction occurs spontaneously, or the case is promptly treated. A majority of the patients probably recover without difficulty, but, as in other dislocations, a displacement may recur. The danger is from inflammatory processes being aroused by the imprudent or excessive use of the articulation directly after the injury, and I believe that quite serious trouble can follow such neglect or carelessness. Case $\mathrm{V}$, in which a localized synovitis was apparently present, illustrates this, and the cases reported by Mr. Hutchinson have apparently led him to the same conclusion.

The treatment of these cases is easy. The movements of the patient, the manipulation of friends, or the examination of the surgeon with reference to diagnosis, often render further trouble about reduction unnecessary. It is, however, usually accomplished without difficuly by first semi-flexing the elbow, in order to relax the bicepital tendon, and approximate as closely as possible the displaced ligament and the edye of the radial head. Then with the forearm grasped by the left hand, supinate steadily with the right, while the left thumb over the radial head pushes in a backward direction. With a slight click or thud, the obstacle to supination suddenly yields, the head is felt to slip back into place, and the inovements of the joint become normal.

In a recent article ${ }^{6}$ it is claimed that flexion of the elbow to, or a little beyond, a right angle combined with full pronation of the forearm, is an easier and surer way to reduce the displacement; for the part of the radial head opposed to the curve of the orbicular ligament during pronation, is its thin rounded edge, over which the ligament easily slips; while full supination, by presenting a deep edge with a rectangular contour, renders reduction more difficult.

The after-treatment varies according to the symptoms presented. Rest is the most important factor. When slight tenderness, with or without pain, alone exists, as in spontaneously reduced cases, or unreduced ones where the arm has been kept quiet, rest in a sling for twenty-four to forty-eight hours prevents subsequent trouble. Here the displacement was probably quite limited, and the injury slight.

In cases complicated by contusions or secondary inflammation, with swelling, marked tenderness, and pain, rest is absolutely necessary. The elbow should be fixed by an internal angular splint, and the subsequent treatment should be that usually employed for sprains or other dislocations, and their possible sequelæ.

In marked subluxations, extension of the elbow should be avoided for at least two weeks after the

s I'Union Medicale, November 23, 1861, Goyrand of Aix.

- British Medical Journal, January 2, 1886. J. Hutehinson, Jr., F. R.C.S. injury, so as to allow the ruptured ligament to unite; otherwise risk of causing a fresh dislocation by the action of the bicepital tendon will be incurred.

It is not improbable that occasionally these dislocations remain permanently unreduced. In such cases, unless fixed by resulting inflammatory changes, one would expect considerable mobility of the radial head. This would account also for a slight abnormal projection met with in adults. Mr. Hutchinson reports two such cases. Lindeman has seen several patients where permanent enlargement of the elbow has resulted.

The cases alluded to at the commencement of this article are the following:

Case I. - aged four, was brought to the Surgical Out-Patient Department of the Carney Hospital with the history that, on the preceding day, while being dragged by the hand, she had suddenly cried out as if in pain, and when released, was unable to use the arm, which was held motionless at the side. Pain followed all attempts at passive or voluntary motion. On the following inorning, the symptoms had disappeared, and when examined at the Hospital, the only trace of an injury was a slight tenderness over the head of the right radius which, two days later, had also vanished.

CASE II. —— aged three, was injured September 12th, 1885. A baby carriage, behind which she was standing, with its post-transverse bar grasped by both outstretched hands, was suddenly jerked away from her. She cried out at once from pain. The arm was seen hanging stiffly by the patient's side, and any attempt at examination or passive motion was resisted. Seen at the Hospital thirty-six hours later, unable or unwilling, on account of pain, to move hand or elbow. The left arm was held by the side with the elbow somewhat flexed, and the hand semipronated. The patient's efforts were apparently directed to prevent motion of the elbow joint. Comparison by inspection with the right elbow gave negative results. By palpitation a slight tenderness over the radial head was found. Passive motion showed the movements of the elbow unimpaired except that of supination whose arc of motion was limited to one-half its normal extent, although the head of the radius apparently rotated freely. Passive motion also caused pain, especially marked during supination. It was also present to a moderate degree during flexion and extension. During examination, while the elbow was held semiflexed, and the writer was trying to ascertain the exact position and arc of rotation of the radial head, the latter was suddenly felt to change its position with a peculiar sensation, resembling that felt during the reduction of a dislocated humerus as the head slides into the glenoid cavity, and at once the articulation recovered its normal extent and freedom of motion. This change occurred during supination, and only a slight amount of force was exerted when the unexpected reduction occurred. The arm was confined in a sling for forty-eight hours, and the patient was then discharged with a perfectly normal joint.

CAsE III. - - aged four, was treated at the Boston City Hospital by Dr. H. L. Burrell, to whose kindness I am indebted for the necessary data for this record. The patient was injured on the preceding evening, while being lifted by the hand from the floor to a chair. The symptoms reported were pain, not localized by the parents, and that motion of the arm was avoided by the patient. No treatment. When 
first seen at the Hospital, eighteen hours after the accident, the above-reported symptoms were present. The arm hung by the side as usual, in the characteristic position. There was no change in the surface or contour of the limb. 'Tenderness over the radial head; supination limited one-third, and accompanied by pain. Other motions of the elbow normal and free. No deviation from the normal anatomical relations of the joint noticed. Reduced unintentionally by supination (elbow extended), while being examined to ascertain the position of the head of the radius. Treatment: sling for forty-eight hours. Discharged well.

CASE IV. - aged six, also a patient of Dr. Burrell, was injured by being seized by the left hand, and swung around. Inability to use the arm caused the mother to suspect an injury to the shoulder. Pain considerable, but not located. No especial treatment. 'Two days later, the father, while "pulling" the arm, felt something surdenly "snap," and the patient's condition was much improved. Seen at the Hospital the following day. Position of arm normal. No deformity or surface change except a slight swelling below the external condyle. There was a slight tenderness and increase of surface temperature at this point. Motion of elbow normal except a slight limitation of supination from muscular resistance. Treatment: internal angular splint, sling. Apparatus removed four days later, and patient discharged. Recovery perfect.

CAsE V. _ _ aged eleven, was a patient of Dr. G. H. Monks, by whose courtesy I saw the case. On October 3d, 1885, the patient fell eight feet between a wharf and a vessel, into the water. Was seized by the hand, and pulled aboard the vessel. He received an injury to the right elbow, either by striking it on a $\log$ used as a "fender." which was floating between the vessel and the wharf, or by a wrench received while being drawn out of the water. He complained of pain, referred to the elbow, tenderness and impairment of motion. The injury was followed by moderate swelling about the joint, with ecclymosis and superficial abrasion on its flexure surface. Treatment : rubbing with liniment. Condition improved. A few days later, after prolonged playing with "clappers," a performance requiring pronation and supination of the hand in rapid succession, the patient became much worse. The pain increased, and motion of the elbow became quite limited. Was first seen by Dr. Monks at the Carney Hospital October 17th, two weeks after the injury. On the flexure surface of the elbow was the fading discoloration of an extravasation in the later stages of absorption, and a few crusts covering a healed abrasion of the skin. Inspection negative, except, perhaps, a slight puffiness under the external condyle. Marked tenderness, and an indistinct "doughy" feel about the head of the radius. Flexion of elbow diminished one-half, extension one-fourth, pronation normal, supination not especially limited, but painful. Diagnosis : subluxation of the radius with contusion, reduced spontaneously and followed by circumscribed periostitis or possibly synovitis, resulting from imprudent or excessive use of the affected part. Treatment : internal angular splint, sling, cold application to articulation. October $19 \mathrm{th}$, the swelling had disappeared, and the tenderness was much diminished. No pain, and arc of motion increased. The patient continued to steadily improve. Apparatus was discontinued five weeks later, and after carrying the arm in a sling for a few weeks longer, he was discharged nearly well. When last seen, January oth, 1886, there was some tenderness over the anterior surface of the radial head when the hand was supinated, and a slight limitation of flexion of the elbow. Pain follows excessive use of the joint, but the patient has no trouble if this is avoided.

CAsE VI, also a patient of Dr. Monks, resembles Case I so closely that a detailed history is unnecessary. The injury occurred in August, 1885, from the patient being dragged along by the hand. Reduction had already taken place when first seen at the Dispensary. A slight tenderness over the head of the radius was the only abnormal condition found, which soon disappeared, and when last seen, the patient was perfectly well.

\section{RECEN'T PROGRESS IN MENTAL DISEASE.}

BY HENRY R. STEDMAN, M.D.

\section{CEREBRAL AND PSYCHICAL DISORDERS ASSOCIATED WITH LOCOMOTOR ATAXIA.}

Several observers have very recently been engaged in working out independently the relations between cerebral disease (structural and functional) and locomotor ataxia.

The fact that symptoms of cerebral disorder as important and varied as vertigo, congestion and epileptiform attacks, aphasia, hemiplegia, etc., are not uncommon in the preataxic stage of tabes led Fournier ${ }^{1}$ to the natural conclusion that psychical disorder might also be present under the same conditions. In corroboration of this view he finds in his notes seventeen cases in which mental complications have appeared in this stage of the disease. He reports as samples three of the cases. One was characterized by mental enfeeblement, loss of energy, fatigue on slight mental exertion, together with dulness and depression. This state lasted about a year, and then suddenly disappeared. A second case developed strange fancies, poor memory and a depressed, morose and hypochondrical state of mind with suicidal ideas. These symptoms also disappeared entirely and gave place to others of the usual tabetic variety. The third case was ushered in by two classes of symptoms, namely, disorders of sight and myoses and mental disturbance, almost identical in kind with that of the first case; mental inactivity and enfeeblement. The psychical symptoms were not of long duration and seemed to yield to specific treatment. Several years later a new set of symptoms more plainly tabetic, supervened, and finally tabes dorsalis of the classic form developed. The transient nature of these attacks will be noticed. They may also be compared pathogenically to the motor manifestations of tabes, such as ocular paralyses, etc., which are not uncommon in the preataxic stage.

In addition to these passing attacks there are also permanent disorders of the intellect in this stage. The tabes in these cases is marked by an assemblage of psychical symptoms resembling ordinary general paralysis, or more accurately that instructive group of cases which M. Fournier has described as pseudo-general paralysis of syphilitic origin. Mental symptoms of this kind, however, do not develop at the very outset, but usually after unmistakable signs of the locomotor

1 L'Encephale t. 4, 1884, p. 611. 